\title{
Los quipus funerarios de Cuspón como patrimonio cultural de la nación
}

Recibido: 21/10/2017

Aprobado: 01/12/2017

Filomeno Zubieta Núñez

Universidad Nacional José Faustino Sánchez Carrión

< filomenozubieta@hotmail.com >

\section{RESUMEN}

El 27 de abril del 2017 el Ministerio de Cultura declaró como Patrimonio Cultural de la Nación al uso ritual de los quipus en el entierro de los difuntos en el pueblo de Cuspón, provincia de Bolognesi (Ancash). Fue el corolario de 21 años de identificación, estudio y valoración de esta expresión cultural. Un primer acercamiento académico a los quipus funerarios se produjo en 1996 y, a lo largo de los ańos posteriores, estos se acrecentaron y maduraron, hasta -llegado el momento- gestionar ante el Ministerio de Cultura su registro y salvaguarda. Por Resolución No 071-2017-MC el uso ritual de los quipus funerarios en Cuspón son ahora parte del Patrimonio Cultural de la Nación. Con este hito los pobladores de Cuspón refuerzas su identidad cultural y nacional

PALABRAS CLAVE: Quipus funerarios.-Ritos funerarios.-Patrimonio Cultural.-Identidad cultural.

\section{The funerary quipus of Cuspon as national cultural heritage}

\begin{abstract}
On April 27, 2017, the Ministry of Culture declared the ritual use of the quipus in the burial of the deceased in the village of Cuspón, province of Bolognesi (Ancash), as Cultural Heritage of the Nation. It was the corollary of 21 years of identification, study and valuation of this cultural expression. A first academic approach to the funeral quipus took place in 1996 and, over the years thereafter, they increased and matured, until - at the time - to manage before the Ministry of Culture its registration and safeguard. By Resolution No. 071-2017-MC, the ritual use of funerary quipus in Cuspón is now part of the Cultural Heritage of the Nation. With this landmark, the people of Cuspón reinforce their cultural and national identity
\end{abstract}

KEYWORDS: Funerary quipus.-Funerary rites.-Cultural heritage.-Cultural identity. 


\section{Introducción}

${ }_{\mathrm{m}}^{\mathrm{y}}$ uso ritual de los quipus funerarios en el velorio y entierro de los difuntos es una práctica que se manifiesta y pervive en el pueblo de Cuspón, provincia de Bolognesi, Ancash. Acaba de ser declarada como Patrimonio Cultural de la Nación por el Ministerio de Cultura mediante Resolución Viceministerial N.o 071-2017-VMPCIC-MC, con fecha 27 de abril de 2017, publicado en el Diario Oficial El Peruano, el sábado 29 de abril del 2017, el mismo que fue entregado a las autoridades del centro poblado de Cuspón en una ceremonia desarrollada en el Club Ancash el pasado 11 de mayo y en la propia comunidad el 24 de junio. Este es un logro que nos enorgullece y que lo compartimos. ${ }^{1}$

Esta práctica del uso de quipus en los rituales funerarios está relacionada con el final del ciclo vital de las personas, niños o adultos, hombres o mujeres. Todos, al momento de morir, para ser velados y ser sepultados deben portar sus quipus, como salvoconductos para lograr el traslado al más allá. Incluso, si la defunción se produce fuera del pueblo (Chiquián, Lima, etc.).

\section{Material y métodos}

La investigación centró su atención en el seguimiento a todo el proceso de identificación, conocimiento y valoración del uso ritual de los quipus funerarios en Cuspón (Chiquián, Bolognesi, Ancash) hasta su declaratoria como Patrimonio Cultural de la Nación por parte del Ministerio de Cultura, lapso que abarca 21 años de 1996 al 2017. El tipo de investigación básica, no experimental; de nivel descriptivo, exploratorio y explicativo, con un enfoque cualitativo. Para la Población se considera a los estudiosos que abordaron el tema de los quipus funerarios, como a los pobladores de Cuspón que observaron el proceso de conocimiento y valoración hasta su declaratoria como Patrimonio Cultural de la Nación. La Muestra, por tratarse de una investigación cualitativa estuvo compuesta por los estudiosos del artefacto cultural y pobladores de la comunidad de Cuspón que cuantitativamente son pocos pero de enorme importancia cualitativa.

1 Para leer la Resolución respectiva hacer click en:

http://busquedas.elperuano.com.pe/normaslegales/declaranpatrimonio-cultural-de-la-nacion-al-ritual-funerari-resolucion-viceministerial-n-071-2017-vmpcic-mc-1514356-1/).
Las Técnicas de recolección de datos.-A tono con el objetivo propuesto, nuestras actividades estuvieron orientadas a: Trabajo de campo: Recolección de la más amplia información entre los pobladores, la quipucamayoc y sus hijos, en la comunidad de Cuspón. Trabajo de gabinete: Recopilación de la información documental, bibliográfica y hemerográfica sobre el tema; procesar la información recolectada y elaborar los informes, siguiendo las orientaciones recibidas y bajo un marco teórico apropiado. Técnicas para el procesamiento de datos: Análisis de la información recogida. Elaboración del informe final. Formulación de conclusiones.

\section{Resultados}

\section{La comunidad de cuspón y los quipus funerarios}

\section{El centro poblado de Cuspón}

El centro poblado de Cuspón es uno de los caseríos del distrito de Chiquián, provincia de Bolognesi, departamento de Ancash. Es también una comunidad campesina, reconocida tempranamente en mayo de 1929, lo que fue posible porque Cuspón conservó los títulos de propiedad de sus ayllus originarios reconocidos en 1612.

Limita con los centros poblados colindantes de Chiquián, Llaclla, Pacllón, Tauripón, Ticllos y Roca.

Unos quince centros arqueológicos dispersos en sus predios son muestra de la antigüedad de la presencia humana en la zona. En algunos de ellos, como en los aledaños a Matara, se encuentran hasta siete variedades de la cantuta, ofreciendo un paisaje multicolor a la vista de los visitantes.

Como caserío tiene a las parcialidades de Matara, Mataracocha y Huancacocha.

Está ubicado geográficamente en la cuenca alta del valle de Pativilca, manteniéndose sobre una economía de subsistencia basada en la agricultura y la ganadería, complementadas con una producción artesanal y un comercio mínimo, en un paisaje escarpado que históricamente ha dificultado la comunicación de esta comunidad con el exterior. Las carreteras a Llaclla y a Roca, producto del esfuerzo mancomunado de sus pobladores, han ayudado a superar en los últimos años este aislamiento. 
En épocas recientes, el fenómeno de la migración del campo a la ciudad ha determinado una disminución de su población y, actualmente, sólo viven de modo permanente en el espacio de la comunidad y sus anexos o parcialidades alrededor de 150 habitantes.

A sus centros arqueológicos se suman otras expresiones culturales en gastronomía, mitos y leyendas, música, danza, además de sus singulares costumbres y festividades. En esta última destaca la fiesta en honor a san Luis, patrono del pueblo, que tiene como día central el 05 de noviembre donde se presentan estampas que recuerdan el encuentro de Francisco Pizarro con Atahualpa, con los denominados capitán e inca, acompańados de pallas, como de bandas y orquestas de música, licores y viandas típicas.

Dentro de sus prácticas ancestrales encontramos la pervivencia del uso ritual de los quipus funerarios en el velatorio y entierro de sus difuntos.

\section{Los quipus funerarios en la historia de Cuspón}

La historia de los quipus funerarios de Cuspón tiene sus raíces en el pueblo colonial de San Luis de Matara que desapareciera hacia fines del siglo XviII por una peste, explicada en la leyenda de Pisana María. Sus pobladores se trasladaron a Chiquián y algunos a sus tierras de las partes bajas llevando sus santos (san Luis y san Pedro) y sus costumbres, como los quipus para el entierro de sus difuntos, dando origen al nuevo pueblo de San Luis de Cuspón (Zubieta, 2003, pp. 13-20). El entierro de los difuntos con el quipu funerario fue una práctica de todos los pueblos de la Doctrina de San Francisco de Chiquián y se mantiene en muchos otros. El centro poblado de Cuspón y su parcialidad de Matara aun la conserva y práctica. Igual ocurre en los pueblos de Roca y Ticllos y parcialmente en los pueblos de Canis, Llipa, Cajamarquilla y otros. En algunos, como Chiquián y Llaclla, ya no se practica.

Los quipus son entendidos como «dispositivos de cuerdas anudadas...elaborados con fibras de algodón o de camélido (llamas y alpacas), hilados y plegados... Las cuerdas se anudan comúnmente en patrones complejos, con nudos a diferentes niveles a lo largo de las longitudes de las llamadas cuerdas «colgantes». Desde las primeras décadas del siglo $\mathrm{xx}$ se sabe que muchos de los cordeles con nudos registraron valores numéricos en el sistema de base 10 de recuento, utilizado por los administradores de habla quechua del estado Inca. Las cuerdas de quipus son a menudo, muy coloridas, y se piensa que los colores significaban las identidades (por ejemplo, nombres, clases o categorías, etc.) de los objetos registrados en estos dispositivos (Urton, 2014, p. 9). Su uso de generaliza con los incas, habiendo aparecido mucho más antes.

Es más, «registraban información sobre la cantidad de vasallos del Imperio, habitantes de cada pueblo, ingresos y salidas de almacenes, tributos de los indios en especie o trabajo así como cantidad de tierras asignadas (topos)»(Wong \& Salcedo, 2005, p. 34).

Estudios posteriores constataron que los quipus prehispánicos no solo eran instrumentos contables y que, durante, la dominación colonial española su uso no solo continuó, sino que se diversificó acorde con la interpretación y usanza que le fueron dando. Como lo expresara el estudioso Frank Salomon (www.casadelcorregidor.pe/tertulias/tertulia_Salomon.php): «el arte de los 'quipus' no murió durante la Colonia (siglos XVI al XIX), como comúnmente se opina, sino que duró hasta tiempos modernos». Así aparecen -entre otroslos llamados quipus funerarios que perviven hasta nuestros días.

Los quipus funerarios, para nuestro caso, son un producto sincrético. Sintetiza las prácticas ancestrales de registros contables de la actividad agropecuaria y mercantil con la presencia de los religiosos españoles y su labor evangelizadora. Las reducciones que progresivamente se fueron estableciendo conllevan la presencia de los sacerdotes para afirmar la presencia colonial española. A los quipus de contar se agregaron los quipus de enterrar, al decir de la quipucamayoc Mama Licuna.

Las prendas utilizadas por los indígenas para sepultar a sus difuntos fueron adoptadas y adaptadas de la vestimenta de los sacerdotes. La vestimenta sacerdotal se reducía a las siguientes prendas: el alba, enterizo similar a un camisón hasta cerca de los talones; las sandalias que tenían cierto parecido a los llanques que usaban los indígenas; el cíngulo, cordón o cinta de seda o de lino, con una borla a cada extremo, que el sacerdote se ciñe el alba desde la cintura. Para sepultar a sus difuntos, el alba fue convertida en mortaja a base de bayeta o tela burda de lana de llama u oveja para cubrir el cuerpo, el cíngulo fue convertido en quipu funerario con nudos y cruces en los extremos y para su larga caminata hacia el más allá se les puso unas sandalias. Con el tiempo, esto fue variando, la mortaja de bayeta fue reemplazada en algunos casos por vestimenta moderna, se elaboraron sandalias de cartones y se mantuvieron los quipus funerarios con las características descritas. 
Progresivamente se convirtió en una práctica de la vida cotidiana del pueblo y su uso se fue transmitiendo y conservando de generación en generación. Las mujeres por su dedicación más cercana a los enfermos y difuntos, convirtieron la elaboración del quipu en un quehacer de las mujeres más ancianas, las más indicadas para la preservación de la memoria local. Así, se fue transmitiendo de madre a hija, como ocurre hasta nuestros días.

\section{Breve descripción del uso ritual de los quipus funerarios}

\section{La preparación del quipu funerario}

Cuando se produce la muerte de un poblador, niño o adulto, hombre o mujer, de inmediato se anuncia a la población con el repique de la campana de la iglesia. La familia prepara el velorio. Las mujeres ancianas bañan al difunto o difunta. En la sala más grande de la casa se coloca una mesa lo suficientemente grande como para habilitar una especie de cama para el difunto(a), con sábanas de bayeta. Antes se vestía al difunto (a) con un hábito de bayeta con capucha y hasta cerca de los talones, hoy algunos visten con el mejor de su ropaje, si es nuevo mejor. Se le coloca sandalias de cartón sostenidas con hilos.

Entretanto en el patio, la quipucamayoc recibe de la familia del occiso las madejas de hilos (blanco y negro) y va preparando el quipu, con torceles y nudos. Ya terminado, entre cánticos y rezos coloca el quipu al cuerpo que ya se halla sobre la mesa de velación.

Para la confección del quipu funerario se requiere de unos 16 o 17 metros de lana hilada, (unas 14 brazadas) dependiendo un poco de la altura de la persona. Va amarrado en la cintura y las puntas a la punta de los pies, colgados tres nudos en la pierna derecha y otro con cuatro nudos, en la izquierda. Cada extremo termina en una cruz.

Los nudos y las cruces son oraciones. Los siete nudos son siete «avemarías» y las cruces son dos «padrenuestros». Las "avemarías» constituyen un mensaje del difunto dirigido a la Virgen María para que ella proteja a su alma y le dé apoyo para que en su traslado de un lado al otro. Similares súplicas, aunque dirigidas a Dios, contienen los dos «padrenuestros» con sus siete peticiones.

\section{La muerte y los rituales de entierro.}

Los quipus funerarios cumplen la función de ser una ayuda, un apoyo o salvoconducto para que el alma vaya superando los obstáculos (simbolizados por perros negros -diablos-), para que la virgen María y Dios escuchen sus oraciones, lo protejan y pueda llegar al cielo.

Los pobladores van llegando al velatorio, muchos llevan víveres. La familia debe desollar por lo menos un buen toro para tener carne para los días del velorio y entierro, pues en esos días todos comen en la casa del difunto. El velorio dura dos días y el entierro se produce al tercero. Se acompaña en el velorio todo el tiempo, día noche. Con comida y licores. En las noches se consume el "chinguirito» o calentado. Es costumbre el narrar cuentos de aparecidos, de «almas», «diablos», etc., así como realizar algunos juegos con castigos corporales para los perdedores, como «esconde la correa».

El carpintero del pueblo va preparando el ataúd. El tercer día, muy temprano, un grupo de varones va al cementerio a abrir la zanja donde reposarán los restos del difunto (a), acompañado de bebidas y comida. Se debe dejar todo listo. El sepelio debe ir acompañado de una banda músicos, por lo general vienen del vecino pueblo de Ticllos. A falta del sacerdote, se contrata los servicios de una persona entendida en cánticos y rezos, llamado "cantor», que oficia durante el velorio, desplazamiento y entierro.

Recién para trasladar al difunto a su última morada se introduce el cadáver en el ataúd, previamente arreglado con frazadas y algunos objetos muy queridos del difunto (a). El difunto porta su quipu. El cortejo fúnebre se dirige primero a la iglesia, con paradas, rezos y cánticos. De la iglesia se dirige al cementerio siempre con sus velas y ofrendas florales. Entre cánticos, llantos y rezos de da el entierro. Al final, hacia la cabeza, se coloca una cruz de madera con las referencias del difunto (a).

La mesa de velorio con las prendas más cercanas continúa velándose dos días más, hasta el quinto día. El quinto día, llamado Piskay, es el día señalado para que todos los pobladores vayan a casa del difunto y de allí a la aguada, llevando todas las prendas de vestir, como frazadas y prendas que haya usado el difunto (a) susceptibles de ser lavadas. Con gran cantidad de quinua remojada en costales se prepara el detergente para lavar las ropas, frazadas, pellejos, etc. de uso del 
difunto. Aquí se producen juegos, incluso bruscos, con los golpes que se dan entre hombres y mujeres con los pellejos y frazadas mojadas. Todo limpio es trasladado a la casa del difunto. Entretanto, la quinua lavada es dispuesta para la mazamorra que se distribuye en ollas y baldes (para la casa) entre todos los que participaron en la faena. Con el "Quinto Día» terminan los funerales. Por lo general, en la entrada general, portón o zaguán se coloca una cruz negra de telas, señal de duelo. El luto, el vestido negro, se lleva durante un ańo. $\mathrm{Al}$ ańo, ante la tumba del pariente, el esposo o esposa, se quita el luto.

Los quipus y la continuidad de una herencia sociocultural

Los quipus funerarios de Cuspón son únicos en su elaboración por mujeres. Forman parte de un capital de experiencia manual y tecnológica manejable y transmitido de madre a hija.

Los casos documentados de mujeres quipucamayoc o quipus hechos por mujeres son escasos. Lo conocemos solo en base a las fuentes orales.

La más cercana es la historia de vida de dońa Gregoria Rivera Zubieta, más conocida como Mama Licuna que cumplía la función de quipucamayoc en el pueblo de Cuspón; falleció el 14 de octubre de 2014.

Aparte de la Mama Licuna, su hija y sus predecesoras, existieron otras mujeres quipucamayoc en tiempos actuales, aunque no se descarta que los quipucamayoc sean hombres, como lo evidencia la presencia de Cipriano Carhuachín Rivera, «Shipico», hijo de Mama Licuna.

Respecto a Mama Licuna, se conoce que ella vio sus quipus como una forma de mantener vivas las tradiciones e ideologías de su pueblo, compartiendo obligaciones con todos los miembros de la comunidad, sin gozar de prerrogativas especiales por este trabajo de crear quipus y de preservar las tradiciones andinas.

Hoy en Cuspón se asegura la continuidad en la manufactura de los quipus por los hijos de Mama Licuna: Elisa y Cipriano. Su práctica lo asume la familia como una forma de continuidad de la identidad comunal.

El reconocimiento legal que realiza el Ministerio de Cultural como parte del como Patrimonio Cultural de la Nación, permitirá que los pobladores de Cuspón se sienten en la obligación de mantenerlo y cuidarlo como parte de su memoria andina contemporánea en el Perú actual.

\section{Los quipus y la valoración de las expresiones culturales de cuspón}

\section{Estudios y logros en torno a los quipus y otras expresiones culturales}

A fines de julio de 1996 viajamos a Cuspón con el Dr. Arturo Ruiz Estrada, arqueólogo y docente de la Universidad Nacional Mayor de San Marcos, y se tuvo un primer acercamiento con las potencialidades culturales de Cuspón. Con el guiado de don Guillermo Rivera se identificaron 15 sitios arqueológicos prehispánicos e incluso hispánicos, el bosque de cantutas de Matara y se tuvo un primer encuentro con dońa Gregoria Rivera Zubieta, "Mama Licuna» y los quipus funerarios.

Producto de esta y otras visitas el Dr. Ruiz Estrada publicó en 1998, en el Boletín del Museo de Arqueología y Antropología de la Universidad Nacional Mayor de San Marcos, No 8, su estudio, "Los quipus funerarios de Cuspón». Presenta un primer expediente ante el Instituto Nacional de Cultura (No 2074-1996) solicitando la «Defensa del Patrimonio Arqueológico de la Comunidad de Cuspón, Chiquiàn». Paralelamente, el primer alcalde del centro poblado, Sr. Oliver Ríos Malqui, emite la Resolución Municipal declarando como «Zona Intangible» a los 15 sitios arqueológicos identificados por el Dr. Ruiz Estrada e inicia las gestiones ante la Municipalidad Provincial de Bolognesi y el INC de Huaraz para su registro y amparo. En el 2003 publica «Antiguas Ocupaciones Humanas en Cuspón, provincia de Bolognesi» en el compendio Arqueología de la sierra de Ancash de Bebel Ibarra Asencios, estudio que también fue incluido en el libro Cuspón: Comunidad e Identidad (2003) con el título de «El poblamiento prehispánico de Cuspón».

Luego de otras visitas menores, pero significativas por sus proyecciones, en el 2004 se conformó un equipo de investigación denominado los «ccantununas» (aludiendo a la flor de la cantuta de la zona y a los hombres que la visitaban) conformado por: Filomeno Zubieta Núñez, historiador, profesor de la Universidad Nacional José Faustino Sánchez Carrión (organizador); Arturo Ruiz Estrada, arqueólogo, profesor de la Universidad Nacional Mayor de San Marcos; Roberto Aldave Palacios, publicista y promotor de turismo, director de Ecoaventura; Augusto Escalante Apaèstegui, literato, profesor de la Universidad Nacional José Faustino Sánchez Carrión; Narciso Robles Atachagua, 
periodista, conductor de programa folclórico en Radio Agricultura; Linder Ramírez Viena, biólogo, profesor de la Universidad Nacional José Faustino Sánchez Carrión.

Los días del 3 al 9 de abril de 2004 se realizaron los estudios multidisciplinarios. Con apoyo de la comunidad que proveyó de acémilas, refrigerio y guiado, se recorrieron sus principales parajes. Aquí fue importante el papel de "Ishico», Isidoro Zubieta Vicuña, el guía, por su amplio conocimiento del entorno geográfico, sus recursos y las historias que cada uno de ellos encerraba. Fueron días de mucho aprendizaje y emociones.

Los resultados fueron progresivos. Roberto Aldave Palacios perfiló y dio cuerpo al documental "Por la Ruta de la Cantuta», un proyecto turístico con Cuspón como principal centro atractivo por sus vestigios arqueológicos, su bosque de cantutas de siete colores, sus quipus funerarios y "Mama Licuna", sus platos típicos y la hospitalidad de su gente. Arturo Ruiz Estrada desarrolló un proyecto mayor sobre los quipus funerarios en la Universidad Nacional Mayor de San Marcos. Linder Ramírez, luego de varias visitas más a Cuspón con sus alumnos, culminó su estudio sobre la flora de Cuspón y Chiquián, dando continuidad al trabajo pionero de Enma Cerrate Valenzuela. Narciso Robles Atachagua, escribió poemas a Cuspón y dedicó muchos de sus programas radiales a ponderar las potencialidades del medio. Por su lado, Augusto Escalante elaboró muchas composiciones que fueron llevadas al canto por Carlos Oro Lázaro y que hoy los escucharemos, además es autor del poema «Buenos Días Chiquián», publicado mucho antes que saliera el programa radial del buen Vladimiro Reyes, además del libro de poemas «Luis Pardo, para llevarte siempre».

Las posteriores visitas, algunas por separado, tanto de Roberto Aldave, como de Arturo Ruiz Estrada, nos han permitido tener una visión de conjunto del patrimonio cultural (arqueológico, histórico y turístico) del distrito de Chiquián, condensados en el libro Chiquián: Arqueología, Identidad y Turismo que, gracias al auspicio de la Municipalidad Provincial de Bolognesi, publicamos en el 2008. La participación del Dr. Arturo Ruiz Estrada y del literato Augusto Escalante Apaéstegui en los eventos de la Asociación de Poetas y Escritores de Ancash, AEPA, con ponencias sobre el entorno chiquiano, como en el XVII Encuentro en homenaje a legendario Luis Pardo, los días 3 al 5 de enero 2009, es el correlato de todas estas visitas.
En una de las tertulias de la Academia Nacional de la Historia, el Dr. Federico Kauffmann Doig (entonces Embajador del Perú en Alemania), mostró su interés por los quipus funerarios $y$, nos reiteró una y otra vez, «tenemos que ir a Cuspón». Luego de muchas tentativas, se concretó la visita los días 26, 27, 28 y 29 de agosto de 2009. Nos acompañó la historiadora de arte, Dra. Alba Choque Porras.

Doña Gregoria Rivera Zubieta, "Mama Licuna», con esa buena predisposición que le era característica, luego de cambiarse de vestimenta adecuada, recibió a los visitantes en la entrada de su casa, acompañada por su hijo «Shipico» (Cipriano Carhuachín Rivera). El encuentro con el Dr. Kauffmann fue emocionante. En quechua Mama Licuna fue narrando y describiendo la confección, características y significado de los quipus, «Shipico» se encargó de la traducción correspondiente. Es más, sobre una mesa con Shipico simulando a un difunto, con buen sentido didáctico, hizo la demostración cómo lo portan los difuntos el quipu en su velatorio y entierro. Al final Mama Licuna le entregó un quipu al Dr. Kauffmann para su feliz viaje llegado el momento.

Poco después una página entera en el diario $E l$ Comercio daría cuenta de este viaje, en la pluma de la periodista Gabriela Machuca con un título sugerente: La última quipucamayoc. Por su lado Alba Choque Porras, con motivo del fallecimiento de Mama Licuna, ofreció elaborar un documental en su homenaje, esperemos se haga realidad.

A estos viajes le sucedieron muchos más con el propósito de complementar la información recogida o trasladar a turistas interesados en lo cultural o vivencial. Aquí hay que destacar la labor de Roberto Aldave Palacios quien organizó visitas con estudiosos como Carlos Milla Villena, Rafo León e interesó a divulgadores como Alfonsina Barrionuevo, Canal 7, El Comercio, etc. Elaboró su propuesta de Circuito Turístico «La Ruta de la Cantuta» incluyendo a Chiquián-Conococha-Cueva de Shagshamay-TicllosRoca-Cuspón-Matara-Chiquián. Su documental del mismo nombre fue reelaborado y mejorado para finalmente quedar como "Llamanaani por la ruta de la flor de la cantuta», que fue presentado en Washington, USA, en la semana "Kaypi Perú» que en castellano significa "Esto es mi Perú», desarrollado entre el 29 de julio al 3 de agosto del 2014, en el Smithsonian National Museum of the American Indian de Washington, DC; igualmente en el Congreso de la República del Perú, 
coincidentemente el 14 de octubre de 2014, día del fallecimiento de Mama Licuna.

El interés por los estudios sobre nuestros quipus funerarios trascendieron las fronteras del Perú. En junio del 2015 la etnomatemática Molly Anne Tun obtuvo su doctorado PhD en Filosofía en la Universidad de Minessota de Estados Unidos con la Tesis: «El quipu: escritura andina en las redes informáticas incaicas y coloniales», dedicándole un capítulo ìntegro a los quipus funerarios de Cuspón. Es más, con ella preparamos un estudio con el título «Los quipus funerarios y tributarios de Cuspön y Chiquiän: Hoy y ayer" publicado en la revista Arqueología y Sociedad del Museo de Arqueología de la Universidad Nacional Mayor de San Marcos. Igualmente con la historiadora polaca Magdalena Setlak se publicó el estudio: «La noción de "camino» en los hilos. Una aproximación a los quipus funerarios de Cuspón (Áncash, Perú)» en Anales del Museo Nacional de Antropología de Madrid, 2017.

Y, gracias a esta Resolución del Ministerio de Cultura, tenemos la certeza que el interés y los estudios se acrecentarán.

\section{Afirmando la identidad cultural de Cuspón}

Todo lo anterior provocó una serie de interrogantes entre los pobladores de Cuspón. ¿Por qué venían estudiosos a sus tierras? ¿Qué de singular tenía Cuspón para interesar a extrańos? Los cambios fueron progresivos y notables. Poco a poco fueron tomando conciencia de las potencialidades en historia, costumbres, gastronomía, patrimonio arqueológico y todo lo que tenían. Se fue construyendo y afirmando una identidad cultural, elevando la autoestima personal y colectiva. Muchos de sus hijos o descendientes se sumaron a divulgar sus atractivos turísticos y culturales: Marco Calderón Ríos y Adrían Carhuachín Lázaro con fotografías a través de las redes; Lucio Rivera Zubieta y Genaro Lázaro Ríos unieron esfuerzos y hoy dirigen un programa radial semanal en Lima, bajo el nombre «Por la ruta de la cantuta»; igual labor realizan en Chiquián Flor Durán Jaimes y Germán Perfecto Hipolo desde su emisora Radio Satélite.

Que una expresión cultural de un pequeño pueblo, caserío del distrito de Chiquián en la provincia de Bolognesi, Ancash, haya logrado su declaratoria como Patrimonio Cultural de Nación alegra a todos. Por muchas razones: todos nuestros pueblos tienen enormes recursos y potencialidades naturales y culturales que deben ser identificados, estudiados, conocidos y valorados. Esperemos que este paso que se acaba de dar abra un camino mayor hacia la revaloración de todas nuestras expresiones culturales.

\section{Conclusiones}

Los resultados de la investigación realizada sobre el uso de los quipus en los rituales funerarios en Cuspón y su declaratoria como Patrimonio Cultural de la Nación por el Ministerio de Cultura motivan algunas reflexiones a manera de conclusiones:

El uso de los quipus en los rituales funerarios en Cuspón es una práctica muy antigua, es un producto sincrético. Se fue formando y afirmando durante los tiempos de la evangelización española arrastrando prácticas prehispánicas con aditamentos de los religiosos. Paralelamente a los "quipus de contar» se mantuvo a lo largo de los siglos. Una práctica extendida a muchos pueblos se redujo y solo se mantiene en Cuspón, aun cuando es conocido su uso en los pueblos vecinos.

En los últimas décadas se mantuvo gracias a las quipucamayoc mujeres, siendo la última doña Gregoria Rivera Zubieta que falleciera el 14 de octubre de 2014, teniendo como continuadores a sus hijos Cipriano y Elisa Carhuachín Rivera.

A partir de 1996, con la visita del Dr. Arturo Ruiz Estrada, los «quipus funerarios» se van dando a conocer en el mundo académico. Una nueva visita multidisciplinaria de investigadores en 2004 refuerza el proceso de su conocimiento y valoración a través de informaciones periodísticas, publicaciones temáticas y difusión de documentales. A partir del 2009, con la visita del Dr. Federico Kauffmann Doig y la presentación en diversos escenarios del país y del extranjero del documental «Llamanaani, por la ruta de la flor de la cantuta» de Roberto Aldave Palacios en que se presenta a la quipucamayoc de Cuspón, se refuerza y consolida. Nuevos estudios y publicaciones se dan conocer.

El 4 de marzo del 2016 se presenta el expediente dirigido al Ministerio de Cultura solicitando se declare como Patrimonio Cultural de la Nación. Finalmente, el 27 de abril del 2017 se expide la Resolución Viceministerial No 071-2017-VMPCIC-MC del Ministerio de Cultura, por el cual se dispone en su 
Art. $1^{\circ}$ «Declarar Patrimonio Cultural de la Nación al Ritual funerario de Cuspón del distrito de Chiquián, provincia de Bolognesi, región Ancash, en el que destaca el uso de quipus funerarios, en tanto se trata de una práctica cultural en la que confluyen la ritualidad católica, una narrativa oral sobre el mundo espiritual, una compleja red de relaciones sociales y la original interpretación y uso de quipus para la protección del difunto en su viaje al más allá».

La declaratoria como Patrimonio Cultural de la Nación al uso ritual de los quipus funerarios de Cuspón contribuye a afirmar la identidad cultural de los pobladores de Cuspón, a elevar su autoestima y los compromete a salvaguardar la vigencia de esta expresión cultural.

Lo ocurrido con los quipus funerarios de Cuspón es un estímulo para que otras expresiones culturales de otros pueblos, distritos, provincias y departamentos, sean identificados, estudiados, valorados y salvaguardados como integrantes del Patrimonio Cultural de la Nación.

\section{Fuentes de información}

Machuca, Gabriela (2009). La última quipucamayoc. Visita del Dr. Federico Kauffmann a Cuspón. El Comercio, 12 de setiembre de 2009. Recuperado de: http://naloalvaradochiquian.blogspot. pe/2009/09/cientifico-federico-kauffmann-doigen.html

Ministerio de Cultura (2014). Manual sobre declaratorias de expresiones del Patrimonio Cultural Inmaterial como Patrimonio Cultural de la Nación. Lima: Ministerio de Cultura. 32 pp.

Ministerio de Cultura (27, 04, 2017). Resolución No 071-2017 declarando Patrimonio Cultural de la Nación al Uso Funerario de los Quipus de Cuspón. Recuperado de: http://busquedas.elperuano.com. pe/normaslegales/declaran-patrimonio-culturalde-la-nacion-al-ritual-funerari-resolucion-viceministerial-n-071-2017-vmpcic-mc-1514356-1/

Ruiz Estrada, Arturo (1998). Los quipus funerarios de Cuspón. Boletín del Museo de Arqueología y Antropología. Universidad Nacional Mayor de San Marcos. No. 8, pp. 12-13 y 18.

Ruiz Estrada, Arturo (2003). «Antiguas ocupaciones humanas en Cuspón: Provincia de Bolognesi». Arqueología de la sierra de Ancash, propuestas y perspectivas (Bebel Herrera Asencios, editor). pp. 405-416.

Ruiz Estrada, Arturo (2008). Patrimonio arqueológico de Chiquián. Chiquián: arqueología, identidad y turismo. Huacho, Perú: Gráfica Imagen. pp. 9-50.

SAlomon, Frank (2010). Tertulia: grafismo andino. (18.07.2010). Reproducido de: www. casadelcorregidor.pe/tertulias/tertulia_Salomon. php

Setlak, Magdalena \& Zubieta, Filomeno (2017). La noción de «camino» en los hilos. Una aproximación a los quipus funerarios de Cuspón (Áncash, Perú). Anales del Museo Nacional de Antropología. No 18. (Ejemplar dedicado a: Etnografías de la muerte en América Latina). Madrid, Espańa, págs. 96-108

Tun, Molly A. (2015). El quipu: Escritura andina en las redes informaticas incaicas y coloniales (Order No. 3727855). Available from ProQuest Dissertations \& Theses Global. (1734030392). Tesis de doctorado. Universidad de Minnesota, USA.

Tun, Molly \& Zubieta Núńez, Filomeno (2017). Los quipus tributarios y funerarios de Cuspón y Chiquián, hoy y ayer. Revista Arqueología y Sociedad. No 31. Museo de Arqueología de la Universidad Nacional Mayor de San Marcos, pp. 403-421.

Urton, Gary (2014). Quipus de Pachacamac. Lima: Ministerio de Cultura. 80 pp.

Wong, Zelma \& Salcedo, Luisa (2005). Quipu: nudos numéricos y parlantes. Kipukamayoc. Vol. 12. No 24, pp. 33-38).

Zubieta NúNEEZ, Filomeno (2003). Cuspón: comunidad e identidad. Huacho, Perú: Gráfica Imagen. 110 pp.

Zubieta NúŃEz, Filomeno (2008a). Chiquián: Cultura e identidad. Chiquián: arqueología, identidad y turismo. Huacho, Perú: Gráfica Imagen. pp. 51-106.

Zubieta NúÑEz, Filomeno (2008b). Sobre identidad, turismo vivencial y educación. Entrevista de Virginia Vílchez de www,librosperuanos.com, febrero de 2008. Recuperado: http://www.librosperuanos. com/autores/articulo/00000001207/ Sobre-identidad-turis mo-vivencial-yeducacionEntrevista-a-Filomeno-Zubieta

Zubieta NúÑEZ, Filomeno (2014). $85^{\circ}$ Aniversario de la Comunidad Campesina de Cuspón (mayo de 2014). Recuperado de http://www.ancashnoticias. com/detalle-noticia.php?id=cultura.3906 
Zubieta NúŃEz, Filomeno (2015). Al año de la muerte de Mama Licuna. Octubre de 2015. Recuperado de http://naloalvaradochiquian.blogspot. pe/2015/10/a-un-ano-de-la-muerte-de-mamalicuna.html
Zubieta NúŃEz, Filomeno (2016). Expediente No 6189393, conteniendo el Oficio No 05-2016-FZN del 11 de marzo de 2016 dirigido a la Ministra de Cultura, solicitando se declare como Patrimonio Cultural de la Nación al Uso Ritual de los Quipus Funerarios de Cuspón. 170 folios. 


\section{ANEXO}

Declaran Patrimonio Cultural de la Nación al Ritual funerario de Cuspón del distrito de Chiquián, provincia de Bolognesi, región Áncash

Resolución viceministerial No 071-2017-vmpcic-mc

Lima, 27 de abril de 2017

Vistos, el Oficio $\mathrm{N}^{\circ}$ 05-2016-FZN suscrito por el señor Filomeno Zubieta Núńez, el Informe No 000093-2017/DPI/DGPC/VMPCIC/MC de la Dirección de Patrimonio Inmaterial, y el Informe $\mathrm{N}^{\circ}$ 000264-2017/DGPC/VMPCIC/MC de la Dirección General de Patrimonio Cultural; y,

CONSIDERANDO:

Que, el primer párrafo del artículo 21 de la Constitución Política del Perú señala que los yacimientos y restos arqueológicos, construcciones, monumentos, lugares, documentos bibliográficos y de archivo, objetos artísticos y testimonios de valor histórico, expresamente declarados bienes culturales, y provisionalmente los que se presumen como tales, son Patrimonio Cultural de la Nación, independientemente de su condición de propiedad privada o pública, los mismos que se encuentran protegidos por el Estado;

Que, el inciso 1 del artículo 2 de la Convención para la Salvaguardia del Patrimonio Cultural Inmaterial de la UNESCO, establece que «se entiende por Patrimonio Cultural Inmaterial los usos, representaciones, expresiones, conocimientos y técnicas - junto con los instrumentos, objetos, artefactos y espacios culturales que les son inherentes - que las comunidades, los grupos y en algunos casos los individuos reconozcan como parte integrante de su patrimonio cultural. Este patrimonio cultural inmaterial que se trasmite de generación en generación es recreado constantemente por las comunidades y grupos en función de su entorno, su interacción con la naturaleza y su historia, infundiéndoles un sentimiento de identidad y continuidad, y contribuyendo así a promover el respeto de la diversidad cultural y la creatividad humana»;

Que, el numeral 2) del artículo 1 de la Ley No 28296, Ley General del Patrimonio Cultural de la Nación, señala que «Integran el Patrimonio Inmaterial de la Nación las creaciones de una comunidad cultural fundadas en las tradiciones, expresadas por individuos de manera unitaria o grupal, y que reconocidamente responden a las expectativas de la comunidad, como expresión de la identidad cultural y social, además de los valores transmitidos oralmente, tales como los idiomas, lenguas y dialectos autóctonos, el saber y conocimiento tradicional, ya sean artísticos, gastronómicos, medicinales, tecnológicos, folclóricos o religiosos, los conocimientos colectivos de los pueblos y otras expresiones o manifestaciones culturales que en conjunto conforman nuestra diversidad cultural»;

Que, el literal b) del artículo 7 de la Ley N ${ }^{\circ} 29565$, Ley de creación del Ministerio de Cultura, modificado por el Decreto Legislativo $\mathrm{N}^{\circ} 1255$, establece que es función exclusiva del Ministerio de Cultura «Realizar acciones de declaración, generación de catastro, delimitación, actualización catastral, investigación, protección, conservación, puesta en valor, promoción y difusión del Patrimonio Cultural de la Nación»;

Que, el artículo 55 del Reglamento de Organización y Funciones del Ministerio de Cultura, aprobado mediante Decreto Supremo $N^{\circ}$ 005-2013-MC establece que «La Dirección de Patrimonio Inmaterial es la unidad orgánica encargada de gestionar, identificar, documentar, registrar, inventariar, investigar, preservar, salvaguardar, promover, valorizar, transmitir $y$ revalorizar el patrimonio cultural inmaterial del país, en sus distintos aspectos, promoviendo la participación activa de la comunidad, los grupos o individuos que crean, mantienen y transmiten dicho patrimonio y de asociarlos activamente en la gestión del mismo. Depende jerárquicamente de la Dirección General de Patrimonio Cultural»;

Que, mediante Acta de Sesión Extraordinaria del Concejo Municipal del Centro Poblado de Cuspón, de fecha 20 de febrero de 2016, se acuerda presentar ante el Ministerio de Cultura, la solicitud de declaratoria como Patrimonio Cultural Inmaterial de la Nación, el uso de los quipus funerarios en el entierro de sus difuntos;

Que, mediante Oficio No 05-2016-FZN del 11 de marzo de 2016, el señor Filomeno Zubieta Núñez (en adelante el administrado), solicitó que se declare Patrimonio Cultural de la Nación el Uso ritual de los quipus funerarios en Cuspón;

Que, mediante Informe No 000264-2017/DGPC/ VMPCIC/MC del 3 abril de 2017, la Dirección 
General de Patrimonio Cultural hace suyo el Informe $\mathrm{N}^{\circ}$ 000093-2017/DPI/DGPC/VMPCIC/MC de la misma fecha, emitido por la Dirección de Patrimonio Inmaterial, por el que se recomienda declarar como Patrimonio Cultural de la Nación al Ritual funerario de Cuspón, en el que destaca el uso de los quipus funerarios;

Que, según el Informe $\mathrm{N}^{\circ}$ 000093-2017/DPI/ DGPC/VMPCIC/MC del 3 de abril de 2017, la Dirección de Patrimonio Inmaterial emite las siguientes precisiones:

Que, el centro poblado de Cuspón, del distrito de Chiquián, provincia de Bolognesi, departamento de Ancash, es también una comunidad campesina, reconocida tempranamente en mayo de 1929, lo que fue posible porque Cuspón conservó los títulos de propiedad de sus ayllus originarios reconocidos en 1612. Limitando con los Centros Poblados colindantes de Chiquián, Llaclla, Pacclón, Tauripón, Ticllos y Roca, y teniendo como parcialidades a los caseríos de Matara, Mataracocha y Huancacocha, Cuspón está ubicado geográficamente en la cuenca alta del valle de Pativilca, manteniéndose sobre una economía de subsistencia basada en la agricultura y la ganadería, complementadas con una producción artesanal y un comercio mínimo, en un paisaje escarpado que históricamente ha dificultado la comunicación de esta comunidad con el exterior. Las carreteras a Llaclla y a Roca han ayudado a superar recientemente este aislamiento. Unos quince centros arqueológicos dispersos en sus predios son muestra de la antigüedad de la presencia humana en la zona. En épocas recientes, el fenómeno de la migración del campo a la ciudad ha determinado una disminución de su población y, actualmente sólo viven de modo permanente en el espacio de la comunidad y sus anexos alrededor de 150 habitantes;

Que, el uso de los quipus funerarios constituye una práctica excepcional, tanto por la escasez de ejemplos paralelos como por la modalidad en que se presenta. El quipu elaborado en Cuspón es parte de los rituales fúnebres que desde tiempos antiguos se han practicado en el distrito de Chiquián, provincia de Bolognesi;

Que, al anuncio de un fallecimiento con repique de campanas de la iglesia local, se prepara el velorio de la persona fallecida, cuyo cuerpo será lavado por mujeres de edad, vestido y puesto en una mesa a modo de cama, cubierto con sábanas de bayeta. Se viste al difunto con su mejor ropa, pero en cambio en sus pies se colocan simples sandalias de cartón sostenidas con hilos;

Que, como parte de la costumbre, el fallecido debe ser velado y enterrado con un quipu elaborado especialmente para esta ocasión. En Cuspón el quipu es una pieza hecha de unas 14 brazadas (de 16 a 17 metros) de lana blanca y negra entrelazada, en cuyos extremos son hechos una serie de nudos. El quipu resultante, de unos 6 metros de largo, es amarrado a la cintura del difunto antes de su velación. Los extremos del quipu son atados a ambos pies del difunto, llevando tres nudos en el tramo que va sobre la pierna derecha y cuatro nudos en la izquierda. Ambos extremos rematan en una cruz. Los siete nudos hechos sobre el quipu y las dos cruces en ambos remates indican las veces que deben ser rezadas, respectivamente, las oraciones conocidas como el Ave María y el Padre Nuestro. Con estas oraciones, que suelen ser pronunciadas por la persona quipucamayoq, se invoca a la Virgen María y a Dios Padre - el ruego incluye siete peticiones - para la protección del alma del difunto en su paso al otro mundo, en cuyo trayecto puede ser asaltado por el demonio, representado en la forma de perros negros. Es decir, estos quipus son leídos a la manera de un rosario que indica qué oraciones deben ser dadas y el número de veces que han de ser dichas durante el trayecto del difunto hacia el otro mundo. Diversos relatos orales indican que, sin el quipu, el alma del difunto no podrá descansar;

Que, durante los dos o tres días en que se desarrolla el velorio y el día del entierro se recibe a las visitas con comida y bebida, parte de ella proporcionada por los mismos comensales en contribución. La bebida preparada para la ocasión es el chinguirito o calentado. Durante el velorio es costumbre contar historias sobre aparecidos, almas errantes y el diablo, y realizar algunos juegos dentro de un ambiente de camaradería. Al tercer día, se irá al camposanto donde los asistentes varones ayudarán a abrir la zanja y a colocar al difunto en el ataúd. La comitiva va acompañada por una banda de músicos y por un cantor local para los oficios durante el velorio y el entierro. El difunto es llevado con sus artículos más queridos, arreglos florales, una cruz de madera con las referencias al fallecido, y se coloca ante la fosa, rodeado de cirios, antes de proceder al entierro;

Que, el velorio continuará en cuerpo ausente en los días siguientes, con la ropa del difunto, hasta el quinto día, llamado piskay, (cinco, en quechua) en que todas las prendas del difunto son lavadas al aire libre 
con el agua o lavaza en la que previamente se ha lavado la quinua que será usada para preparar una mazamorra que consumirán los colaboradores. Con ello se dan por terminados los funerales. El uso de ropa negra en señal de luto seguirá para los familiares o cónyuges por un año; de tratarse de un cónyuge, el luto de su pareja terminará formalmente al cambiar ésta de ropa ante la tumba del difunto;

Que, con relación al quipu, cabe señalar que este es un sistema de registro de información originario de tiempos prehispánicos, aparecido al menos hacia finales del primer milenio de nuestra era, correspondiente al Horizonte Medio, en que se produjo una notable expansión urbana. Este sistema llegó a la cumbre de su utilización como parte del extenso y complejo sistema de administración del Tawantinsuyu, que requería de un constante registro de recursos disponibles y de censo poblacional, para la regulación fiscal, el monto de tributos y la organización de la fuerza de trabajo acorde a las necesidades concretas del Estado. Toda esta información era registrada con este sistema de cordeles y nudos, manejada por un tipo especial de funcionario conocido como quipucamayoq;

Que, el quipu incaico era un sistema complejo de registro que consistía en un cordel principal al cual iban atados otros cordeles subsidiarios; en los quipus que registraban cantidades los cordeles subsidiarios tenían nudos cuyo significado numeral se indicaba por el número de vueltas que los componían, y los números eran registrados sobre un sistema de posicionamiento decimal, en unidades, decenas, centenas, millares e incluso decenas de millares; de no haber una cantidad que registrar en algún nivel se dejaba un espacio vacío. El quipu era leído por el código de nudos y por los colores del cordel, lo que según cronistas y estudiosos hacía referencia al sujeto a contar. El cordel principal podía presentar a su vez una serie de nudos que representaban una sumatoria de los cordeles secundarios. Pero además, según relatan las crónicas con insistencia, se usaba el quipu como recurso nemotécnico de textos narrativos, ya se trate de relatos históricos, de genealogías e incluso de textos líricos. El código con el que tales quipus deben haberse leído no fue registrado por ningún documento escrito y por ello es desconocido;

Que, el uso del quipu decreció con el establecimiento del virreinato y de sus sistemas de registro escritos, y una gran parte de esta información fue destruida físicamente en el proceso de Conquista, quedando actualmente ejemplares obtenidos de entierros, en su casi totalidad de carácter numeral. Pero el uso del quipu permaneció entre la población rural indígena en diversas regiones, para el registro de población y de recursos disponibles, distribuidos en unidades organizadas como barrios o parcialidades, herederas de la organización dual propia del mundo andino. De este modo, los quipus se mantuvieron en diversos puntos bajo el sistema colonial, y sobrevivieron dentro del régimen de haciendas, en comunidades independientes durante el período republicano, dispersos en áreas de Cusco, Puno, Huancavelica, La Libertad, Ancash o Lima;

Que, la difusión del sistema escolar en zonas como la sierra central, donde el uso del quipu era importante, junto con las necesidades impuestas por el reconocimiento legal de las comunidades ante el Estado, motivó la sustitución del sistema de registro por nudos por un sistema escrito, perdiéndose el recuerdo del código original de lectura de estos quipus y reforzando de este modo la marginalización de una de las máximas creaciones de la civilización andina. En respuesta, el uso práctico de los quipus dio lugar a un uso ritual del objeto, incluso sobre quipus elaborados originalmente para la contabilidad de gentes o recursos. De este modo, en la comunidad de San Andrés de Tupicocha (Huarochirí, Lima) una serie de quipus antiguos es llevada a modo de emblema por las autoridades de las parcialidades que conforman la comunidad, durante las ceremonias de cambio de autoridades. El quipu de Rapaz (Oyón, Lima), un ejemplar de notable amplitud, que lleva atados diversos objetos junto con los nudos, es conservado como una reliquia por las autoridades para un ritual propiciador del agua. El quipu funerario de Cuspón, de carácter igualmente ritual, es producto de la historia particular de la región donde se ha desarrollado;

Que, la elaboración y uso del quipu funerario de Cuspón deriva de la historia de las costumbres funerarias del distrito, en la que están presentes elementos nativos e hispanos. El registro histórico de Cuspón refiere que la población nativa de la región fue originalmente ubicada en el pueblo de San Luis de Matara, cuya población fue diezmada por una peste, al parecer de viruela negra; esto produce el abandono del pueblo y la emigración de la población superviviente a San Francisco de Chiquián, como cabeza de doctrina, y a Cuspón, reducción de indios que formaba parte de la misma doctrina. Los pobladores trasladaron a 
Chiquián muchas imágenes y a Cuspón las de San Pedro y San Luis, este último es desde entonces el patrono de esta comunidad. Con la migración se difundieron las costumbres originarias de este lugar, entre las cuales estaba su particular tradición funeraria;

Que, en Cuspón el quipu funerario forma parte del ajuar del difunto, quien era vestido con prendas que derivaban del hábito sacerdotal cristiano. Antiguamente se vestía al difunto con un hábito con capucha llamado alba, derivado del hábito sacerdotal, hecho de bayeta y que llegaba a los tobillos. Se le colocaban sandalias, fácilmente asociadas al calzado cerrado andino conocido como llanque, y el cíngulo, un cordón de seda o lino con una borla a cada extremo, usado para ceńirse el alba a la cintura. El cinturón del traje sacerdotal de diversas órdenes religiosas es un largo cordel al cual se le hacían nudos que indicaban, a modo de un rosario de rezos, el número y tipo de oraciones que el sacerdote debía de hacer durante su oficio religioso. Del alba derivó la mortaja con que se vestía al difunto, y como parte de ella el cinturón para sujetar la túnica mortuoria llevaba una serie de nudos, lo que ha sido asociado al sistema de registro conocido como quipu, nombre que mantuvo esta prenda. Este ha sido el elemento que ha sobrevivido en las prácticas funerarias, al abandonarse la mortaja original por una vestimenta de uso cotidiano del difunto. El quipu de Cuspón es de este modo a la vez un rosario y un amuleto; la serie de nudos que deberán ser leídos, al invocar a María y al Dios cristiano, permitirá al difunto superar los obstáculos en su camino al paraíso;

Que, sería inexacto, sin embargo, considerar esta una costumbre de origen exclusivamente hispano. Una parte de los quipus arqueológicos que se conservan hoy en día pertenecen a entierros, siendo colocados en el fardo funerario como una prenda más, de lo que puede deducirse que en ciertas regiones esta fue una práctica extendida, a pesar de no contar con referencias del significado de esta costumbre. Aunque técnicamente el quipu de Cuspón sea leído como un rosario, presenta algunas características que lo emparentan con el quipu andino. Una de ellas es la conformación de los nudos, similar a la de los nudos de los quipus incaicos. De igual modo, se ve un vínculo en la elaboración del cordón con hilos de dos colores - en este caso uno blanco y otro de tono oscuro, sea negro, marrón o azul- que también se ha encontrado en algunos quipus antiguos. Finalmente, entre las coincidencias más notorias, cabe señalar el nombre de quipucamayoq dado a las personas especializadas en su elaboración;

Que, el expediente menciona que el uso de quipus funerarios era extendido en toda la doctrina de Chiquián en tiempos del virreinato y ha seguido practicándose en diversos centros poblados. Aparte del más conocido, Cuspón, lugar donde este tipo de quipu aún se elabora, se sigue practicando esta costumbre en los pueblos circunvecinos de la provincia de Bolognesi, como Roca y Ticllos del distrito de Ticllos; Canis, Llipa y Cajamarquilla, de los distritos de Corpanqui y Canis, y se mantuvo hasta hace algún tiempo no determinado, en los pueblos de Chiquián y Llaclla, capital del distrito de Abelardo Pardo Lezameta. Lo que hace único al centro poblado de Cuspón es que es el único lugar en que estos quipus siguen siendo elaborados;

Que, el conocimiento de su elaboración y lectura es transmitido de madres a hijas, aunque en la práctica su fabricación, al requerir de varios metros de hilo de lana, se hace con el apoyo de otros miembros de la familia, de ambos sexos. En Cuspón esta ha sido la potestad de una línea de descendencia de mujeres de una familia, cuyo miembro reciente más notorio fue la seńora Gregoria Rivera Zubieta, conocida como Mama Licuna. Durante su vida fue la única quipucamayoq de Cuspón y, en general, de la provincia de Bolognesi, hasta su fallecimiento en el año 2014. Doña Gregoria clasificaba a los quipus en dos grupos: los quipus para contar y los quipus para enterrar, en los que se especializó, según propia declaración, para mantener viva la tradición local, sin reclamar ninguna prerrogativa especial por su contribución a esta causa. Su papel ha sido heredado por sus hijos Cipriano y Elisa Carhuachín Rivera, quienes mantienen el conocimiento y los contenidos asociados a esta práctica, aprendidos de su madre;

Que, la elaboración y uso de quipus es parte fundamental de los ritos funerarios de Cuspón, con la particularidad de que en esta comunidad campesina estos son elaborados por un linaje de detentadores de esta tradición. Sus características formales difieren parcialmente de las del quipu prehispánico porque es producto de una historia particular del cristianismo en los Andes, siendo una interpretación nativa de un ritual católico;

Que, conjuntamente con las referencias históricas, el Informe $\mathrm{N}^{\circ}$ 000093-2017/DPI/DGPC/VMPCIC/ MC de la Dirección de Patrimonio Inmaterial detalla las características, importancia, valor, alcance 
y significados del Ritual funerario de Cuspón, en el que destaca el uso de los quipus funerarios, motivo por el cual constituye parte integrante de la presente Resolución, conforme a lo dispuesto en el artículo 6 del Texto Único Ordenado de la Ley $N^{\circ}$ 27444, Ley del Procedimiento Administrativo General, aprobada mediante Decreto Supremo N 006-2017-JUS;

Que, mediante Resolución Ministerial $N^{\circ} 338$ 2015-MC, se aprobó la Directiva Nº03-2015-MC, «Declaratoria de las manifestaciones de Patrimonio Cultural Inmaterial y de la obra de grandes maestros, sabios y creadores como Patrimonio Cultural de la Nación y Declaratoria de Interés Cultural», en la que se establecen los lineamientos y normas para la tramitación del expediente de declaratoria de Patrimonio Cultural de la Nación de las manifestaciones de patrimonio cultural inmaterial, correspondiendo al Viceministerio de Patrimonio Cultural e Industrias Culturales declarar las manifestaciones del Patrimonio Cultural Inmaterial como Patrimonio Cultural de la Nación;

De conformidad con lo establecido en la Constitución Política del Perú; en la Ley N²8296, Ley General del Patrimonio Cultural de la Nación; en la Ley No 29565, Ley de Creación del Ministerio de Cultura; en el Reglamento de la Ley General de Patrimonio Cultural de la Nación, aprobado por Decreto Supremo No 011-2006-ED; en el Reglamento de Organización y Funciones del Ministerio de Cultura, aprobado por Decreto Supremo No 005-2013-MC; y la Directiva No 003-2015-MC, aprobada por Resolución Ministerial No 338-2015-MC;

\section{SE RESUELVE:}

Artículo 1.- Declarar Patrimonio Cultural de la Nación al Ritual funerario de Cuspón del distrito de Chiquián, provincia de Bolognesi, región Ancash, en el que destaca el uso de quipus funerarios, en tanto se trata de una práctica cultural en la que confluyen la ritualidad católica, una narrativa oral sobre el mundo espiritual, una compleja red de relaciones sociales y la original interpretación y uso de quipus para la protección del difunto en su viaje al más allá.

Artículo 2.- Disponer la publicación de la presente Resolución en el Diario Oficial El Peruano y la difusión del Informe $N^{\circ}$ 000093-2017/DPI/DGPC/VMPCIC/ MC y la presente Resolución en el Portal Institucional del Ministerio de Cultura (www.cultura.gob.pe).

Artículo 3.- Encargar a la Dirección de Patrimonio Inmaterial, en conjunto con la Dirección Desconcentrada de Cultura de Ancash, la elaboración cada cinco (5) años de un informe detallado sobre el estado de la expresión declarada, de modo que su registro institucional pueda ser actualizado en cuanto a los cambios producidos en la manifestación, los riesgos que pudiesen surgir para su vigencia, y otros aspectos relevantes para realizar un seguimiento institucional de su desenvolvimiento y salvaguardia, de ser el caso.

Artículo 4.- Notificar la presente Resolución y el Informe $\mathrm{N}^{\circ}$ 000093-2017/DPI/DGPC/VMPCIC/ MC a la Dirección Desconcentrada de Cultura de Ancash, a la Municipalidad Provincial de Bolognesi, al Municipio del Centro Poblado de Cuspón, a la Junta Directiva de la Comunidad Campesina de Cuspón y al señor Filomeno Zubieta Núñez para los fines consiguientes.

Regístrese, comuníquese y publíquese.

JORGE ERNESTO ARRUNATEGUI GADEA

Viceministro de Patrimonio Cultural e Industrias Culturales.

\section{Fuente:}

MINISTERIO DE CULTURA DEL PERÚ (27, 04, 2017). Resolución No 071-2017 declarando Patrimonio Cultural de la Nación al Uso Funerario de los Quipus de Cuspón. Recuperado de: http:// busquedas.elperuano.com.pe/normaslegales/declaranpatrimonio-cultural-de-la-nacion-al-ritual-funerariresolucion-vice-ministerial-n-071-2017-vmpcicmc-1514356-1/ 